\title{
Many-body depletion interactions among protein spheres in a semidilute polymer solution
}

\author{
Theo Odijk \\ Faculty of Chemical Engineering and Materials Science, Delft University of Technology, P.O. Box 5045, \\ 2600 GA Delft, the Netherlands
}

(Received 11 June 1996; accepted 22 November 1996)

\begin{abstract}
The polymer segment distribution is shown to obey the Laplace equation for a suspension of small protein spheres and semidilute polymer. The depletion interaction is computed at all protein concentrations by introducing void distributions. Within a linear response Ansatz for the solution to the Laplace equation, the average depletion energy depends on two- and three-point void (-surface) correlation functions. It is concluded that depletion correlations of long range do not appear at high protein concentrations. (C) 1997 American Institute of Physics. [S0021-9606(97)51108-1]
\end{abstract}

\section{INTRODUCTION}

The effective interaction among particles in a mixture is a subject in its own right. It has been known for a long time that the elimination of certain degrees of freedom gives rise to nontrivial potentials of mean force ${ }^{1}$ that are nonpairwiseadditive. In the past, little analytical work appears to have been carried out to comprehend the statistical mechanics of this type of interaction. ${ }^{2}$ One approach due to Fixman is to expand the potential of mean force in terms of density fluctuations around the actual average density instead of around density zero. ${ }^{3}$ This ruse could well be exploited in problems involving mixtures if the fluctuations are small enough in some sense. More recently there have been efforts to address the interactions in mixtures of colloids and polymers. ${ }^{4-10}$ These systems have the theoretical advantage of being strongly asymmetric, at least potentially.

Here, our purpose is to discuss one type of systemprotein-polymer mixtures-where formal headway with respect to nonpairwise-additive interactions can be made without undue mathematical complications. Globular proteins are often substantially smaller than the typical correlation length of the surrounding polymeric medium which results in an essential simplification of the statistical mechanics. De Gennes $^{11}$ and, recently, the author ${ }^{12}$ noted that various physical quantities may be readily understood for a single small sphere immersed in a semidilute polymer solution, in terms of straightforward though nontrivial scaling relations. One ingredient is the basic validity of the Laplace equation ${ }^{12}$ for the distribution of the polymer segments. This notion will be extended here for spheres at all concentrations up to close packing. Accordingly, the protein-polymer problem in this limit is quite similar to the mathematics of diffusion in a random array of spheres, an analogy that will be exploited here.

Within a rough zero-order approximation, globular proteins may often be viewed as inert small impenetrable spheres. This approximation obviously has its drawbacks for it is known that proteins at their isoelectric point exhibit weak attractive forces in aqueous solution. ${ }^{13-17}$ The scope of this paper is restricted to an analysis of many-body polymerprotein interactions accounting for the nonuniformity of the segment distribution of the flexible polymers. The consideration of phase separation is left to future work.

This paper differs from previous theoretical work ${ }^{4-10}$ for a method is developed to deal with the statistical mechanics of a mixture at all levels of the many-body correlations. First, it is shown that the polymer distribution satisfies a Laplace equation to a high degree of accuracy. Hence, a simple free energy functional involves merely a gradient of the polymer density. The depletion of the polymer at the protein surfaces may be related to a suitable void probe function via a response function, at least in an attempt correct to the linear order. Thus, ultimately, minimization of the average energy functional should yield the response in terms of void correlations. The averaging of both polymer and sphere configurations is effected at the same time and this is an essential advantage of the current theory. Our purpose is to compute the depletion interaction as a function of the protein volume fraction almost up to close packing.

\section{VALIDITY OF THE LAPLACE EQUATION}

In a semidilute solution of long flexible polymers, the statistical properties of the system are independent of the polymer contour length. Hence, the segment density is a relevant variable. But fluctuations are of crucial importance in the semidilute limit. ${ }^{18}$ Accordingly, the partition function of a mixture of $n$ hard spheres of radius $a$ and entangled polymer chains at semidilute concentrations, may be schematically represented by

$$
Z=\int d \mathbf{r}_{1}, \ldots, d \mathbf{r}_{n} \int \mathscr{D}[c(\mathbf{r})] e^{-U_{\mathrm{tot}} / k_{B} T}
$$

with

$$
\begin{aligned}
U_{\text {tot }}= & U_{h}\left(\mathbf{r}_{1}, \mathbf{r}_{2}, \ldots, \mathbf{r}_{n}\right)+U_{d}\left(\mathbf{r}_{1}, \ldots, \mathbf{r}_{n}, c(\mathbf{r})\right) \\
& +U_{p}[c(\mathbf{r})] .
\end{aligned}
$$

Here, $k_{B}$, is Boltzmann's constant and $T$ is the temperature. Both the hard-sphere and depletion interactions, $U_{h}$ and $U_{d}$, depend on the protein centers of mass denoted by $\mathbf{r}_{1}, \mathbf{r}_{2}, \ldots, \mathbf{r}_{n}$. Furthermore, the interaction $U_{d}$ among the protein particles is also a functional of the concentration $c(\mathbf{r})$ of 
polymer segments. Formally, we also expect a term $U_{p}$ arising from the interaction between polymer segments that are outside some convoluted depletion zone centered on $\left\{\mathbf{r}_{1}, \ldots, \mathbf{r}_{n}\right\}$. I suppose the flexible polymer is well soluble in the solvent (water). The dependence of the protein-polymer interaction on the quality of the solvent is subtle ${ }^{12}$ but is neglected here. The general form Eq. (1) exhibiting a concentration measure $\mathscr{\mathscr { L }}[c(\mathbf{r})]$, is shown explicitly here in order to stress two potential hurdles in the resolution of the manybody problem: (a) the fluctuations in the segment concentration; (b) elimination of the polymer degrees of freedom will lead to an interaction which could be strongly nonpairwiseadditive at high protein concentrations. Fortunately, the first problem is greatly simplified in the limit of small spheres, as I now show.

First, we momentarily forget about segment fluctuations and simplify Eq. (1) in a self-consistent field approximation. Then, $U_{p}$ is simply a constant and $U_{d}$ is known explicitly. ${ }^{19}$ At a fixed configuration of proteins, $U_{d}$ in the integrand of Eq. (1) has a minimum when $c(\mathbf{r})$ equals $c_{m}(\mathbf{r}) \equiv c_{0} \varphi^{2}(\mathbf{r})$ given by

$$
\Delta^{1} \varphi+\left(\frac{a^{2}}{\xi^{2}}\right) g(\varphi)=0 .
$$

The Laplacian is denoted by $\Delta$; all coordinates are conveniently scaled by $a: \mathbf{r}^{1} \equiv \mathbf{r} / a$. The average segment concentration is $c_{0}$; in solution there will exist voids completely free from proteins, with a uniform polymer concentration $c_{v} \neq c_{0}$ since the volume fraction $v$ of protein spheres is nonzero. Equation (3) is simply the usual self-consistent field equation $^{19}$ for $\varphi=\varphi\left(\mathbf{r}^{1}, \mathbf{r}_{1}^{1}, \ldots, \mathbf{r}_{n}^{1}\right)$, with a correlation length $\xi=\xi\left(c_{v}\right)$. Joanny et al. ${ }^{19}$ have discussed the eigenvalue equation (3) in some detail and have analyzed the resulting depletion near a planar wall. The dimensionless function $g$ has the form $\varphi-\varphi^{m}$ with $m>1$. The boundary conditions on $\varphi$ are (a) $\varphi=0$ at the surface of the spheres; (b) $\varphi \rightarrow\left(c_{v} / c_{0}\right)^{1 / 2}$ at some point within a void far removed from all the spheres.

Now let the correlation length $\xi\left(c_{v}\right)$ be much greater than $a$. Then, Eq. (3) reduces to the Laplace equation correct to the second order in the small parameter $a / \xi$

$$
\Delta \varphi \simeq 0 \text {. }
$$

However, this "derivation" of Eq. (4) is a bit deceptive for a relevant scale of depletion is implicitly assumed to be $a$. For instance, for a very long rodlike array of aligned spheres, the solution to Eq. (4) is not a uniformly valid approximation to the one given by Eq. (3). Another exception is a large compact cluster of spheres of size much greater than $\xi$ : Depletion beyond the cluster is now poorly described by Eq. (4). On the whole, the validity of the Laplace equation must be based on the occurrence of purported clusters whose maximum span $\lambda$ is, in the main, smaller than $\xi$. Equation (4) can only apply in a statistical sense.

Essentially the same equations (3) and (4) may be written down if one were to use a hybrid theory combining scaling and self-consistent arguments. ${ }^{20}$ In that case, the function $g$ has a different exponent (with again $m>1$ ) but its overall shape remains the same and the correlation length is
$\xi=A^{-5 / 4} c_{v}^{-3 / 4}$, where $A$ is the Kuhn segment length of the polymer. Accordingly, provided $\lambda \ll \xi$, we again conclude that depletion occurs on scales less than the polymer correlation length and its range does not depend on the strength of the interaction between the segments.

We are finally in a position to argue for the essential validity of Eq. (4) even when segment fluctuations are properly taken into account. In the mean-field and hybrid pictures, Eq. (4) is legitimate provided the correlation length is sufficiently large. It is well known that the Laplacian operator results from the square gradient term in the polymer free energy which denotes the ideal entropy of the chains. ${ }^{19}$ In other words, this entropy predominates in depletion when $\xi \gg a$ and segment interactions in some average sense play an insignificant role. But, then, strong fluctuations in these interactions may well modify them into renormalized terms, yet will certainly not enhance them to a level competing with the ideal entropy (recall that the interactions are scaled by a very small coefficient $a^{2} / \xi^{2}$ ). Accordingly, segment fluctuations are unimportant in determining the polymer distribution in the convoluted depletion volume surrounding an array of protein spheres. Within the depletion zone, the segment density obeys Eq. (4). In voids far removed from the spheres, however, segment fluctuations are as important as usual so that a self-consistent field picture will break down there. Hence, we have to use, say, a scaling analysis in order to derive the depletion interaction which is addressed next.

\section{DEPLETION INTERACTION}

It is now argued that the depletion energy has the following form:

$$
U_{d} \simeq\left(\frac{a}{\xi_{0}}\right)^{4 / 3} a^{-1} k_{B} T \int d \mathbf{r}(\nabla \varphi)^{2} .
$$

(a) $U_{d}$ is a function of $a, \mathbf{r}_{1}, \ldots, \mathbf{r}_{n}$ and $c(\mathbf{r})=c_{0} \varphi^{2}(\mathbf{r})$ only; (b) it must be proportional to the number of segments depleted from a convoluted volume surrounding the spheres; this number, in turn is proportional to $c_{0}$ or $\xi_{0}^{-4 / 3}=\xi_{0}^{-4 / 3}\left(c_{0}\right)$; (c) $U_{d}$ is an extensive quantity; (d) Eq. (4) results upon minimization of $U_{d}$ with respect to $\varphi(\mathbf{r})$; (e) Eq. (5) does not have an explicit dependence on $A$ for $\xi_{0}$ is the sole relevant scale of the polymer; (f) $U_{d}$ reduces to the correct expression valid in the limit of infinite dilution of spheres, ${ }^{11} U_{d}$ $\simeq n\left(a / \xi_{0}\right)^{4 / 3} k_{B} T$.

Two general expressions can now be derived. The chemical potential of a polymeric segment is given by

$$
\mu_{p}=-k_{B} T \frac{\partial Z}{Z \partial N}=\mu_{p, 0}+N^{-1}\left\langle U_{d}\right\rangle_{c}
$$

where $N$ is the number of segments in the suspension, $\mu_{p, 0}$ is the chemical potential of a segment in a protein-free solution of concentration equal to that in the protein-free voids, and \langle\rangle$_{c}$ signifies a canonical average. But the osmotic pressure of a semidilute polymer suspension free from protein and of concentration $c_{0}$ is simply ${ }^{18}$ 


$$
\pi_{p, 0} \simeq \frac{k_{B} T}{\xi_{0}^{3}}
$$

Hence, we have a relation between the chemical potential and the osmotic pressure, which may be of experimental relevance

$$
\mu_{p}-\mu_{p, 0} \sim \pi_{p, 0}^{9 / 4} .
$$

The depletion attraction is of intermediate range but is comparatively weak for $a \ll \xi$. One expects the local correlations between spheres to be dominated by their harsh repulsive interactions. Accordingly, the hard-sphere interaction $U_{h}$ may serve as a reference state (index $h$ ). Perturbation theory ${ }^{21}$ gives for the free energy of the spheres

$$
F_{s}=F_{h}+\left\langle U_{d}\right\rangle_{h} .
$$

I next investigate the nature of the average depletion energy $\left\langle U_{d}\right\rangle_{h}$.

\section{NONPAIRWISE-ADDITIVE CORRELATIONS}

Equations (1), (4), (5), and (9) may, of course, be addressed by standard perturbation theories ${ }^{21}$ at low volume fractions $v$ of protein $\left(v \equiv 4 \pi n a^{3} / 3 V ; V=\right.$ total volume of the system). Interactions beyond the pair level may be dealt with by expansions about a nonzero concentration ${ }^{3}$ but I would like to propose a formalism valid, in principle, for all $v$. We have two difficult problems to solve: Eq. (4) and Eq. (9). It would be expedient were we to replace these by one. We first note that the statistical mechanics of the protein-polymer system at hand is a problem in statistical geometry: ${ }^{22} \mathrm{We}$ may view the array of protein spheres as a porous medium with an associated diffusion statement given by Eq. (4). A mixture of large colloids and polymers has also been treated within a porous medium analogy recently. ${ }^{9}$ We might try a numerical analysis of the field $\varphi(\mathbf{r})$ in terms of multipole expansions $\mathrm{s}^{23}$ and then perform the statistical averaging [Eq. (9)]. However, powerful analytic work may proceed in the spirit of classic analyses in the past by Prager ${ }^{24,25}$ and Doi. ${ }^{26}$ It is the polymer distribution in the space $\mathscr{V}$ between the protein spheres that we wish to know; the field $\varphi(\mathbf{r})$ is then logically regarded as a functional of the void probe function ${ }^{27} \eta_{v}(\mathbf{r})$

$\eta_{v}(\mathbf{r})=1 \quad \mathbf{r}$ within $\mathscr{T}$,

$\eta_{v}(\mathbf{r})=0, \quad \mathbf{r}$ within the complement $\overline{\mathscr{V}}(V=\mathscr{V}+\overline{\mathscr{V}})$.

Thus the sphere-sphere correlations themselves are not so interesting but rather the void correlation functions which have been studied at length. ${ }^{22}$ A plausible Ansatz introduced below, is that $\varphi(\mathbf{r})$ responds linearly to $\eta_{v}(\mathbf{r})$. In effect, Eq. (4) is linear in $\varphi(\mathbf{r})$ and $\eta_{v}(\mathbf{r})$ if the boundary condition on the surfaces of the spheres were to be formally incorporated as source terms. In the end, the theory boils down to computing a response function in only one step, which is carried out via a suitable variational principle.

\section{Void correlations}

Before formulating a minimum principle, we first introduce convenient approximate expressions for the void correlation functions. The surface probe function $\eta_{s}(\mathbf{r}) \equiv\left|\boldsymbol{\nabla} \eta_{v}(\mathbf{r})\right|$ is a delta function defined on the surface $S$ of the void. We further define void-void and surface-void correlation functions which reflect the translational invariance of the system.

$$
\begin{gathered}
F_{v v}\left(\mathbf{r}-\mathbf{r}^{\prime}\right) \equiv\left\langle\eta_{v}(\mathbf{r}) \eta_{v}\left(\mathbf{r}^{\prime}\right)\right\rangle_{h}, \\
F_{s v}\left(\mathbf{r}-\mathbf{r}^{\prime}\right) \equiv\left\langle\eta_{s}(\mathbf{r}) \eta_{v}\left(\mathbf{r}^{\prime}\right)\right\rangle_{h} .
\end{gathered}
$$

A convenient approximate form for $F_{v v}$ pertaining to impenetrable spheres is ${ }^{9}$

$$
\begin{aligned}
F_{v v}(\mathbf{t}) & =w^{2}+w v e^{-t / a} \quad t \leqslant 2 a \\
& =w^{2} \quad t>2 a,
\end{aligned}
$$

where $w \equiv 1-v=\left\langle\eta_{v}(\mathbf{r})\right\rangle_{h}$. This has the correct limits at $t=0$ and $t=\infty$ as can be seen from Eq. (11). Equation (13) is fairly close to the correlation determined by Haile et al. ${ }^{28}$ via computer simulations. Note that weak oscillations are clearly present in the numerical work. ${ }^{28}$ Doi $^{26}$ has computed $F_{s v}$ for a certain random model of imbedded spheres which is a popular theoretical artifice for a porous medium

$$
\begin{aligned}
F_{s v}(\mathbf{t}) & =\frac{1}{2} s\left(1+\frac{t}{2 a}\right) w^{-1} F_{v v}(\mathbf{t}) \quad t \leqslant 2 a \\
& =s w \quad t>2 a .
\end{aligned}
$$

It so happens that Eq. (14), but with $F_{v v}$ given by Eq. (13), is a fairly good approximation to the surface-void correlation deduced by computer simulations on impenetrable spheres. ${ }^{29}$ The limits at short and long distances can be inferred from the definition [Eq. (12)]. The average of $\eta_{s}$ is easily calculated because the delta function $\boldsymbol{\nabla} \eta_{v}(\mathbf{r})$ is defined on the surfaces of the spheres

$$
\begin{aligned}
\left\langle\eta_{s}\right\rangle_{h} & =V^{-1} \int d \mathbf{r}\left\langle\eta_{s}(\mathbf{r})\right\rangle_{h} \\
& =V^{-1} \int d S=4 \pi n a^{2} V^{-1}=3 v / a \equiv s .
\end{aligned}
$$

\section{Linear response Ansatz}

Next, I propose a linear response Ansatz ${ }^{24,26}$ as argued above for the function $\varphi(\mathbf{r})$ which is supposed to approximate the solution to the Laplace equation (4)

$$
\varphi(\mathbf{r})=E+\int d \mathbf{r}^{\prime} K\left(\mathbf{r}-\mathbf{r}^{\prime}\right)\left(\eta_{v}\left(\mathbf{r}^{\prime}\right)-w\right) .
$$

This is a continuous function defined throughout the volume $V$ and nonzero within the spheres. The kernel $K$ whose form must reflect the translational invariance of the porous medium after averaging, is to be determined variationally. At very low volume fractions, we expect $K \sim\left|\mathbf{r}-\mathbf{r}^{\prime}\right|^{-1}$ from the solution $^{12}$ to Eq. (4). Hence, the porosity $w$ has been subtracted from $\eta_{v}(\mathbf{r})$ to avoid a potential divergence. Thus the constant $E$ tends to unity ${ }^{12}$ in the limit of vanishing volume fraction. Fortunately, it turns out that the form Eq. (16) leads 
to a renormalizable theory for impenetrable spheres. I have also investigated $\eta_{s}(\mathbf{r})$ as a possible source term but, in that case, formal difficulties appear in the limit $v \rightarrow 0$ which do not seem resolvable.

Depletion implies that $\varphi(\mathbf{r})$ must be identical to zero on $S$. This is evidently difficult to take into account exactly, so we replace this constraint by a global condition which is less stringent

$$
\begin{aligned}
V^{-1} \int d S\langle\varphi(S)\rangle_{h} & =V^{-1} \int d \mathbf{r}\left\langle\eta_{s}(\mathbf{r}) \varphi(\mathbf{r})\right\rangle_{h} \\
& =E s+\int d \mathbf{t} K(\mathbf{t})\left(F_{s v}(\mathbf{t})-w s\right)=0 .
\end{aligned}
$$

A second condition connecting $E$ and $K$ stems from the invariance of the total number of polymer segments in the suspension. On averaging this requirement and taking due care to delete the fictive contribution within the spheres, we have

$$
\begin{aligned}
V^{-1} \int & d \mathbf{r}\left\langle\eta_{v}(\mathbf{r}) \varphi^{\dot{2}}(\mathbf{r})\right\rangle_{h} \\
= & E^{2} w+2 E \int d \mathbf{t} K(\mathbf{t})\left(F_{v v}(\mathbf{t})-w^{2}\right) \\
& +\int d \mathbf{t} \int d \mathbf{u} K(\mathbf{t}) K(\mathbf{u}) H(\mathbf{t}, \mathbf{u})=1
\end{aligned}
$$

with

$$
H(\mathbf{u}, \mathbf{t}) \equiv G_{3}(\mathbf{u}, \mathbf{t})-w F_{v v}(\mathbf{t})-w F_{v v}(\mathbf{u})+w^{3}
$$

and a three-point correlation function

$$
G_{3} \equiv\left\langle\eta_{v}(\mathbf{r}) \eta_{v}\left(\mathbf{r}^{\prime}\right) \eta_{v}\left(\mathbf{r}^{\prime \prime}\right)\right\rangle_{h} \quad\left(\mathbf{t}=\mathbf{r}-\mathbf{r}^{\prime} ; \mathbf{u}=\mathbf{r}-\mathbf{r}^{\prime \prime}\right) .
$$

Note that $E=1$ in the limit $v=0$ as it should.

Despite extensive computations ${ }^{30,31}$ it is not so easy to find a useful uniform approximation to $G_{3}$ valid at all volume fractions. One interesting choice has been discussed by Weissburg and Prager ${ }^{30}$

$$
G_{3} \simeq w^{-1} F_{v v}(\mathbf{a}) F_{v v}(\mathbf{b}),
$$

where $\mathbf{a}$ and $\mathbf{b}$ are the two smallest vectors out of the set $(\mathbf{u}, \mathbf{t}, \mathbf{u}-\mathbf{t})$. At the very least, Eq. (21) has the following merits: It is accurate whenever (i) all three points are well separated $\left(G_{3}=w^{3}\right)$; (ii) $\quad \mathbf{r}^{\prime \prime} \rightarrow \infty \quad$ at fixed $\quad \mathbf{r}$ and $\mathbf{r}^{\prime}\left(G_{3}=w F_{v v}\left(\mathbf{r}-\mathbf{r}^{\prime}\right)\right) ; \quad$ (iii) $\quad \mathbf{r}=\mathbf{r}^{\prime}\left(G_{3}=F_{v v}\left(\mathbf{r}-\mathbf{r}^{\prime \prime}\right)\right)$; (iv) $\mathbf{r}=\mathbf{r}^{\prime}=\mathbf{r}^{\prime \prime}\left(G_{3}=w\right)$. Moreover, it can be readily shown that $H(\mathbf{u}, \mathbf{t}) \geqslant 0$ uniformly, so realistic solutions for $E$ and $K$ are guaranteed. Equation (21) tends to overestimate $G_{3}$ in various regimes, ${ }^{30}$ which causes problems as will be seen below. Still, Eq. (21) is useful in assessing the qualitative nature of the depletion energy as a function of the protein volume fraction.

The constant $E$ is now eliminated to yield a condition on the function $K$. Equations (17)-(21) reduce to

$$
w J^{2}+w^{-1} I_{1}=1,
$$

where

$$
J \equiv \int d \mathbf{t} K(\mathbf{t})\left(w^{-1} F_{v v}(\mathbf{t})-s^{-1} F_{s v}(\mathbf{t})\right)
$$

and

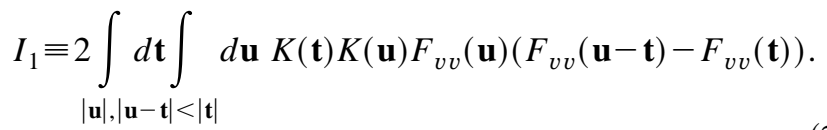

Next, a scaled energy density may be written as

$$
\begin{aligned}
u & \equiv a^{2} V^{-1} \int d \mathbf{r}\left\langle\eta_{v}(\mathbf{r})(\boldsymbol{\nabla} \varphi(\mathbf{r}))^{2}\right\rangle_{h} \\
& =a^{2} \int d \mathbf{t} \int d \mathbf{u} \frac{d K(u)}{d u} \frac{d K(t)}{d t} \frac{\mathbf{t} \cdot \mathbf{u}}{t u} H(\mathbf{u}, \mathbf{t}) .
\end{aligned}
$$

Approximating the three-point correlation by Eq. (21) again, we get

$$
\begin{aligned}
u \simeq & w^{-1} I_{2}, \\
I_{2} \equiv & 2 a^{2} \int_{|\mathbf{u}|,|\mathbf{u}-t|<|\mathbf{t}|} d \mathbf{t} \int_{v v} d \mathbf{u} \frac{d K(u)}{d u} \frac{d K(t)}{d t} \frac{\mathbf{t} \cdot \mathbf{u}}{t u} \\
& \times F_{v v}(\mathbf{u})\left(F_{v v}(\mathbf{u}-\mathbf{t})-F_{v v}(\mathbf{t})\right) .
\end{aligned}
$$

It is now possible to eliminate the scale or $h$ dependence of $K$, i.e., we rescale $h K \Rightarrow K$ employing Eq. (22)

$$
u[K]=\frac{I_{2}}{w^{2} J^{2}+I_{1}} .
$$

This constitutes a minimum principle for $K$.

A plausible choice for $K$ is

$$
K=\frac{e^{-\beta t / a}}{4 \pi a^{2} t} .
$$

The variational parameter $\beta$ is expected to vanish as the volume fraction $v$ of spheres tends to zero; on the other hand, a finite range $a \beta^{-1}$ with $\beta=\mathscr{Q}(1)$ is reasonable as the protein spheres cluster together. If we use Eqs. (13) and (14), the integral $I_{2}$ has the approximate form

$$
\begin{aligned}
I_{2}= & w^{2} v \int_{0}^{2} d z(1+\beta z) e^{-\beta z} \int_{0}^{z} d x(1+\beta x) e^{-\beta x}(w+v x) \\
& \times \int_{x / 2 z}^{1} d y y\left[\exp -\left(x^{2}+z^{2}-2 x z y\right)^{1 / 2}-e^{-z}\right] .
\end{aligned}
$$

Unfortunately, it is now evident from the behavior of the integrand that it contributes substantially to $I_{2}$ in a regime where the approximation Eq. (21) is no longer accurate. ${ }^{30}$ This is explicitly borne out by evaluating the dilute limit $v \ll 1$ : this should yield $u=3 v$ whereas Eqs. (28)-(30) markedly overestimate the numerical coefficient. A better computation of $G_{3}$ at all volume fractions will have to be awaited.

Meanwhile, it is possible to understand the qualitative behavior of the depletion energy. Arguably, the dependence of $G_{3}$ on the volume fraction should be fairly well described by Eq. (21): $G_{3}$ is accurate at several extreme positions. Ac- 
cordingly, the use of a $G_{3}$ similar to Eq. (21) but rescaled to yield the correct numerical coefficient at low protein concentrations, leads to the following estimates:

$$
\begin{aligned}
& I_{1} \simeq \frac{1}{3} \tau\left(1-\alpha_{1} v\right) w^{2} v, \\
& I_{2} \simeq \frac{1}{3} \tau\left(1-\alpha_{2} v\right) w^{2} v
\end{aligned}
$$

with a coefficient $\tau$ close to unity, and $\alpha_{1} \simeq \alpha_{2}$ and $1-\alpha_{1} \ll 1$. Together with the relation

$$
J \simeq \frac{1}{3} w,
$$

these expressions yield

$$
u \simeq \frac{3 \tau\left(1-\alpha_{2} v\right) v}{(1-v)^{2}+3 \tau\left(1-\alpha_{1} v\right) v} .
$$

We conclude that the depletion energy $\left\langle U_{d}\right\rangle_{h}$ $\simeq V\left(a / \xi_{0}\right)^{4 / 3} u a^{-3} k_{B} T$ starts to saturate to a constant value at a protein volume fraction of about $\frac{1}{3}$. Accordingly, this theory does not predict the existence of a second correlation length $\lambda \gg a$; in that case, $U_{d}$ would tend to zero as $v$ approaches the value at close packing. There is no tendency to force the polymer out of clusters of spheres as is seen in simulations of large colloids. ${ }^{8}$ Of course, this arises, in part, from our supposition $a \ll \xi_{0}$. The collective effect of protein crowding is substantial though not overpowering despite the intermediate range of the depletion forces.

\section{CONCLUDING REMARKS}

A general conclusion of this work is that polymerparticle interactions may be markedly mediated by correlations beyond the pair level in qualitative agreement with numerical computations in other regimes. ${ }^{8}$ However, our analysis for small proteins seems to preclude very strong correlations of long range. I have given a treatment at the level of three-point void correlations. A precise computation of these will be needed to gain quantitative understanding of protein-polymer mixtures at all concentrations. It is recalled that in the phase separation of isotropic phases, the protein volume fraction may be quite high. ${ }^{32}$

The present theory accounts for protein-protein correlations up to quite high volume fractions of protein. The effect of segment fluctuations has been proved to be weak for comparatively small protein spheres. Depletion forces arising from the interaction among several proteins and the surrounding sea of polymer segments have been dealt with to a significant degree of correlation. Indeed, the formalism is not at all mean field in the usual sense for delicate three-point correlations have been taken into account. Preaveraging of the depletion constraint as expressed by Eq. (17) is arguably the weakest link in our argumentation.

Note added in proof. The concentration $c_{0}$ of polymer segments refers to the space accessible to the polymer; hence, we have $c_{0} \equiv N / V(1-v)$. The average depletion energy per protein is $\left\langle U_{d}\right\rangle_{h} / n=4 \pi\left(a / \xi_{0}\right)^{4 / 3}(u / v) k_{B} T$ and may sometimes exceed $k_{B} T$; unfortunately, we do not know the precise theoretical value of the numerical prefactor. The depletion energy is quite sensitive to polymer-protein interactions [see T. Odijk, Langmuir (submitted)].

\section{ACKNOWLEDGMENTS}

I thank J. A. M. Smit (University of Leiden) for various discussions. This research is supported by a grant from the programme I.O.P. Eiwitten (Innovative Research Projects: Protein).

${ }^{1}$ W. G. McMillan and J. E. Mayer, J. Chem. Phys. 13, 276 (1945).

${ }^{2}$ H. L. Friedman, A Course in Statistical Mechanics (Prentice Hall, Englewood Cliffs, 1985).

${ }^{3}$ M. Fixman, J. Chem. Phys. 35, 889 (1961)

${ }^{4}$ M. R. Shaw and D. Thirumalai, Phys. Rev. A. 44, R 4797 (1991).

${ }^{5}$ A. Yethiraj, C. K. Hall, and R. Dickman, J. Colloid Int. Sci. 151, 102 (1991).

${ }^{6}$ H. N. W. Lekkerkerker, Colloid Surf. 51, 419 (1991).

${ }^{7}$ H. N. W. Lekkerkerker, W. C. K. Poon, P. N. Pusey, A. Stroobants, and P. B. Warren, Europhys. Lett. 20, 559 (1992).

${ }^{8}$ E. J. Meijer and D. Frenkel, J. Chem. Phys. 100, 6873 (1994).

${ }^{9}$ P. B. Warren, S. M. Ilett, and W. C. K. Poon, Phys. Rev. E 52, 5205 (1995).

${ }^{10}$ W. C. K. Poon and P. N. Pusey, in Observation, Prediction and Simulation of Phase Transitions in Complex Fluids, edited by M. Baus, L. R. Rull, and J. P. Ryckaert (Kluwer Academic, Dordrecht, 1995), p. 3.

${ }^{11}$ P. G. de Gennes, C. R. Acad. Sci. B 288, 359 (1979).

${ }^{12}$ T. Odijk, Macromolecules 29, 1842 (1996).

${ }^{13}$ V. L. Vilker, C. K. Colton, and K. A. Smith, J. Colloid Int. Sci. 79, 548 (1981)

${ }^{14}$ H. M. Schaink, J. A. P. P. van Dijk, and J. A. M. Smit, Internal Report (Unilever/University of Leiden).

${ }^{15}$ A. P. Minton, Biophys. Chem. 57, 65 (1995).

${ }^{16}$ D. Rosenbaum, P. C. Zamora, and C. F. Zukoski, Phys. Rev. Lett. 76, 150 (1996).

${ }^{17}$ A. Lomakin, N. Ashevie, and G. B. Benedek, J. Chem. Phys. 104, 1646 (1996).

${ }^{18}$ J. des Cloizeaux, J. Phys. (Paris) 36, 281 (1975).

${ }^{19}$ J. F. Joanny, L. Leibler, and P. G. de Gennes, J. Polymer Sci. Polymer Phys. 17, 1073 (1979).

${ }^{20}$ P. G. de Gennes, Macromolecules 15, 492 (1982).

${ }^{21}$ J. P. Hansen and I. R. McDonald, Theory of Simple Liquids (Academic, London, 1976).

${ }^{22}$ H. Reiss, J. Phys. Chem. 96, 4736 (1992).

${ }^{23}$ P. Venema and D. Bedeaux, Physica A 156, 835 (1989).

${ }^{24}$ S. Prager, Phys. Fluids 4, 1477 (1961).

${ }^{25}$ S. Prager, Physica 29, 129 (1963).

${ }^{26}$ M. Doi, J. Phys. Soc. Jpn. 40, 567 (1976).

${ }^{27}$ P. Debye, A. R. Anderson, and H. Brumberger, J. Appl. Phys. 28, 679 (1957).

${ }^{28}$ J. M. Haile, C. Massobrio, and S. Torquato, J. Chem. Phys. 83, 4075 (1985).

${ }^{29}$ N. A. Seaton and E. D. Glandt, J. Chem. Phys. 85, 5262 (1986).

${ }^{30}$ H. L. Weissberg and S. Prager, Phys. Fluids 5, 1390 (1962).

${ }^{31}$ S. Torquato and G. Stell, J. Chem. Phys. 82, 980 (1985).

${ }^{32}$ V. B. Tolstoguzov, Food Hydrocolloids 4, 429 (1991). 